\title{
PSEUDO-FERMIONIC COHERENT STATES
}

\author{
OMAR CHERBAL AND MAHREZ DRIR
}

Communicated by Akira Yoshioka

\begin{abstract}
We have generalized the fermionic coherent states to pseudo-fermion oscillator system. The system of coherent states constructed consist of two subsets, which are bi-normalized and bi-overcomplete. The two subsets are built up as eigenstates of two annihilation operators $b$ and $\tilde{b}=\eta b \eta^{-1}$ of respectively $H$ and $H^{+}$where $\eta$ is the Hermitian and invertible operator that ensures the pseudoHermiticity of the Hamiltonian $H=\eta^{-1} H^{+} \eta$.
\end{abstract}

\section{Introduction}

The coherent states which provide a quantum description of the evolution of a classical system [4] has been generalized to several quantum systems [9, 12]. In last years the concept of coherent states was also introduced to non-Hermitian quantum mechanics $[1,10]$. In this perspective, we have constructed in a recent paper [3] pseudo-fermionic coherent states for pseudo-Hermitian two-level Hamiltonians with real spectrum.

Our aim is to develops the ideas of [3] in the case of the single pseudo-fermion or called "phermion" oscillator described by the Hamiltonian $H=\omega\left(b^{\#} b-\frac{1}{2}\right)$. First we start with a review in Section 2 of some main results on the pseudo-Hermiticity. In Section 3 we construct pseudo-fermionic or "phermionic" coherent states for the single phermion oscillator. In Section 4 we study the time evolution of coherent states constructed. The paper ends with concluding remarks.

\section{Some Main Results on Pseudo-Hermiticity}

By definition [5], an Hamiltonian $H$ is called pseudo-Hermitian if it satisfies the relation

$$
H^{+}=\eta H \eta^{-1}
$$


where $\eta$ is a linear, Hermitian, and invertible operator. One can also express the definition (1) in the form

$$
H^{\#}=H
$$

where

$$
H^{\#}=\eta^{-1} H^{+} \eta
$$

is the $\eta$-pseudo adjoint of $H$ [5]. The condition (1) reduces to hermicity when the operator $\eta$ is equal to the identity. The pseudo-Hermitian conjugation \# has the same properties as the Hermitian conjugation ${ }^{+}$, namely

a) $\left(A^{\#}\right)^{\#}=A$

b) $(A B)^{\#}=B^{\#} A^{\#}$

c) $(\alpha A+\beta B)^{\#}=\alpha^{*} A^{\#}+\beta^{*} B^{\#}$, where $A$ and $B$ are linear operators, and $\alpha$ and $\beta$ are complexes numbers.

\section{Pseudo-Fermionic or "Phermionic" Coherent States}

We consider the single pseudo-fermion "phermion" oscillator described by the following Hamiltonian

$$
H=\omega\left(b^{\#} b-\frac{1}{2}\right)
$$

where $\omega$ is constant, $b^{\#}$ and $b$ are respectively the creation and annihilation operators of the single-degree of freedom of what is called the pseudo-Hermitian fermion or a phermion [6], which satisfies the standard anticommutation relations

$$
\left[b, b^{\#}\right]_{+}=b b^{\#}+b^{\#} b=1, \quad(b)^{2}=\left(b^{\#}\right)^{2}=0
$$

$b^{\#}=\eta^{-1} b^{+} \eta$ [5], where $\eta$ is a linear, Hermitian and invertible operator. The phermion number operators is $N=b^{\#} b$ satisfy

$$
[b, N]=b, \quad\left[b^{\#}, N\right]=-b^{\#}, \quad\left[b, b^{\#}\right]=1-2 N .
$$

$H^{+}$satisfies the pseudo-hermiticity relation [5] $\mathrm{H}^{+}=\eta \mathrm{H}^{-1}$. We note that if $\eta=1$, thus $b^{\#}=b^{+}$, the pseudo-Hermitian fermion (phermion) algebra (5) reduces to the usual fermion algebra [6]. By analogy with the Fock space representation of the fermion algebra, the Fock space representation of the phermion algebra is spanned by the two-dimensional simultaneous eigenbasis $\left\{\left|\psi_{1}\right\rangle,\left|\psi_{2}\right\rangle\right\}$ 
of the corresponding number operator $b^{\#} b$. The operators $b$ and $b^{\#}$ allow transitions between the states as

$$
\begin{aligned}
b\left|\psi_{1}\right\rangle & =0, & b\left|\psi_{2}\right\rangle & =\left|\psi_{1}\right\rangle \\
b^{\#}\left|\psi_{2}\right\rangle & =0, & b^{\#}\left|\psi_{1}\right\rangle & =\left|\psi_{2}\right\rangle .
\end{aligned}
$$

The operator $b$ annihilates the lowest eigenstates $\left|\psi_{1}\right\rangle$, and $b^{\#}$ brings this state onto the upper eigenstates $\left|\psi_{2}\right\rangle$.

We define the phermionic coherent states $|\xi\rangle$ in an analogue scheme as the fermionic coherent states $[2,8]$ as follow

$$
|\xi\rangle=\mathrm{e}^{\left(b^{\#} \xi-\xi^{*} b\right)}\left|\psi_{1}\right\rangle=\mathrm{e}^{-\frac{1}{2} \xi^{*} \xi}\left(\left|\psi_{1}\right\rangle-\xi\left|\psi_{2}\right\rangle\right)
$$

where $\xi$ and $\xi^{*}$ are Grassmannian variables which satisfy the anticommutation relations

$$
\left\{\xi, \xi^{*}\right\}=\xi \xi^{*}+\xi^{*} \xi=0, \quad\{\xi, \xi\}=0, \quad\left\{\xi^{*}, \xi^{*}\right\}=0 .
$$

The $\xi$ and $\xi^{*}$ anticommute with $b$ and $b^{\#}$

$$
\begin{array}{rlrl}
\xi b & =-b \xi, \quad \xi^{*} b & =-b \xi^{*} \\
\xi b^{\#} & =-b^{\#} \xi, \quad \xi^{*} b^{\#}=-b^{\#} \xi^{*}
\end{array}
$$

and have the following properties

$$
\begin{aligned}
\xi\left|\psi_{1}\right\rangle & =\left|\psi_{1}\right\rangle \xi, & \xi\left|\psi_{2}\right\rangle & =-\left|\psi_{2}\right\rangle \\
\xi\left|\phi_{1}\right\rangle & =\left|\phi_{1}\right\rangle \xi, & \xi\left|\phi_{2}\right\rangle & =-\left|\phi_{2}\right\rangle \xi
\end{aligned}
$$

The pseudo-Hermitian conjugation reverses the order of all fermionic quantities, both the operators and the Grassmann variables

$$
\left(b^{\#} \xi+\xi^{*} b\right)^{\#}=\xi^{*} b+b^{\#} \xi .
$$

The Grassmann integration and differentiation over the complex Grassmann variables are given by

$$
\begin{aligned}
\int \mathrm{d} \xi 1=0, \quad \int \mathrm{d} \xi \xi=1, \quad \int \mathrm{d} \xi^{*} 1=0, & \int \mathrm{d} \xi^{*} \xi^{*}=1 \\
\frac{\mathrm{d}}{\mathrm{d} \xi} 1=0, \quad \frac{\mathrm{d}}{\mathrm{d} \xi} \xi=1, & \frac{\mathrm{d}}{\mathrm{d} \xi^{*}} 1=0, \quad \frac{\mathrm{d}}{\mathrm{d} \xi^{*}} \xi^{*}=1 .
\end{aligned}
$$


The Grassmann integration of any function is equivalent to the left differentiation

$$
\int \mathrm{d} \xi f(\xi)=\frac{\partial}{\partial \xi} f(\xi) .
$$

The Hermitian adjoint of the coherent state is

$$
\langle\xi|=\mathrm{e}^{-\frac{1}{2} \xi^{*} \xi}\left(\left\langle\psi_{1}\right|+\xi^{*}\left\langle\psi_{2}\right|\right) .
$$

In the same way, we introduce another family of coherent states associated to $\mathrm{H}^{+}$ as follows

$$
\widetilde{|\xi\rangle}=\mathrm{e}^{-\frac{1}{2} \xi^{*} \xi}\left(\left|\phi_{1}\right\rangle-\xi\left|\phi_{2}\right\rangle\right)
$$

where $\left|\phi_{1}\right\rangle$ and $\left|\phi_{2}\right\rangle$ are the eigenstates of $H^{+}$. The Hermitian adjoint of $\widetilde{|\xi\rangle}$ is

$$
\widetilde{\langle\xi|}=\mathrm{e}^{-\frac{1}{2} \xi^{*} \xi}\left(\left\langle\phi_{1}\right|+\xi^{*}\left\langle\phi_{2}\right|\right) .
$$

The scalar product between $\widetilde{\langle\xi|} \widetilde{\xi}\rangle$ takes the form

$$
\widetilde{\langle\xi|} \tilde{\xi}\rangle=\left\langle\phi_{1} \mid \phi_{1}\right\rangle+\left(\left\langle\phi_{2} \mid \phi_{2}\right\rangle-\left\langle\phi_{1} \mid \phi_{1}\right\rangle\right) \xi^{*} \xi-2 \mathrm{i} \operatorname{Im}\left(\xi\left\langle\phi_{1} \mid \phi_{2}\right\rangle\right) \neq 1
$$

while

$$
\widetilde{\langle\xi \mid \xi\rangle}=\left\langle\phi_{1} \mid \psi_{1}\right\rangle+\left(\left\langle\phi_{2} \mid \psi_{2}\right\rangle-\left\langle\phi_{1} \mid \psi_{1}\right\rangle\right) \xi^{*} \xi-2 \mathrm{i} \operatorname{Im}\left(\xi\left\langle\phi_{1} \mid \psi_{2}\right\rangle\right)=1
$$

and

$$
\langle\xi \mid \widetilde{\xi}\rangle=\left\langle\psi_{1} \mid \phi_{1}\right\rangle+\left(\left\langle\psi_{2} \mid \phi_{2}\right\rangle-\left\langle\psi_{1} \mid \phi_{1}\right\rangle\right) \xi^{*} \xi-2 \mathrm{i} \operatorname{Im}\left(\xi\left\langle\psi_{1} \mid \phi_{2}\right\rangle\right)=1
$$

or more generally

$$
\left\langle\xi_{1}\right| \widetilde{\left.\xi_{2}\right\rangle}=\left\langle\psi_{1}\left|D^{+}\left(\xi_{1}\right) \widetilde{D}\left(\xi_{2}\right)\right| \phi_{1}\right\rangle=\xi_{1}^{*} \xi_{2}+\frac{1}{4}\left(2-\xi_{1}^{*} \xi_{1}\right)\left(2-\xi_{2}^{*} \xi_{2}\right) .
$$

By means of the two type of states $|\xi\rangle$ and $\widetilde{|\xi\rangle}$ the resolution of the identity is realized in the following way

$$
1=\int \mathrm{d} \xi^{*} \mathrm{~d} \xi|\xi\rangle \widetilde{\langle\xi|}=\int \mathrm{d} \xi^{*} \mathrm{~d} \xi \widetilde{|\xi\rangle}\langle\xi| .
$$

This leads to the statement: The system of phermionic coherent states $\{|\xi\rangle, \widetilde{|\xi\rangle}\}$ consists of two subsets $\{|\xi\rangle\}$ and $\{\widetilde{|\xi\rangle}\}$, which are bi-normalized and bi-overcomplete.

In the next Section we show that these phermionic coherent states satisfy also the temporal stability property. 


\section{Time Evolution of Phermionic Coherent States}

We study the time evolution of the phermionic coherent states constructed above. We said that the evolution of a given coherent state is time-stable if the time evolution of any initial state from the set, governed by the Hamiltonian, leaves the state in the set for any $t[7,11]$. In the case of our phermionic coherent states $\{|\xi\rangle, \widetilde{\xi}\rangle\}$ the set parameter is the complex Grassmann variable $\xi$, the eigenvalue of the lowering operators $b$ or $\tilde{b}$. The time evolution is stable if the evolved states $|\xi ; t\rangle$ and $\mid \widetilde{\xi ; t\rangle}$ remain eigenstates of the operators $b$ and $\tilde{b}$ respectively

$$
\begin{aligned}
& b|\xi ; t\rangle=\xi(t)|\xi ; t\rangle \\
& \tilde{b}|\widetilde{\xi ; t\rangle}=\xi(t)| \widetilde{\xi ; t\rangle} .
\end{aligned}
$$

This implies that the time evolved coherent states $|\xi ; t\rangle$ and $\mid \widetilde{\xi ; t\rangle}$ should form binormal and bi-overcomlete system. Let us first consider the time evolution of an initial coherent states $|\xi\rangle$. Clearly we have

$$
|\xi ; t\rangle=\mathrm{e}^{(-\mathrm{i} H t)}|\xi\rangle, \quad|\xi ; 0\rangle \equiv|\xi\rangle .
$$

Using the form (9) of $|\xi\rangle$ and the facts that $\left|\psi_{1,2}\right\rangle$ are eigenstates of $H$ (with eigenvalues $E_{1,2}$ ) we get

$$
|\xi ; t\rangle=\mathrm{e}^{-\mathrm{i} E_{1} t}\left(1-\frac{1}{2} \xi^{*} \xi\right)\left|\psi_{1}\right\rangle-\mathrm{e}^{-\mathrm{i} E_{2} t} \xi\left|\psi_{2}\right\rangle .
$$

Taking into account that $E_{1}=-E$ and $E_{2}=E$ we put $\xi(t)=\mathrm{e}^{-\mathrm{i} 2 E t} \xi$ and rewrite the last equation in the form

$$
|\xi ; t\rangle=\mathrm{e}^{\mathrm{i} E t}\left(\left(1-\frac{1}{2} \xi(t)^{*} \xi(t)\right)\left|\psi_{1}\right\rangle-\xi(t)\left|\psi_{2}\right\rangle\right)=\mathrm{e}^{\mathrm{i} E t}|\xi(t)\rangle
$$

which manifests the stability of the time evolution of coherent states $|\xi\rangle$. In a similar manner we establish, that the time evolution $\widetilde{|\xi ; t\rangle}$ of an initial $\widetilde{|\xi\rangle}$, is stable (remains eigenstate of $\tilde{b}$ )

$$
\widetilde{|\xi ; t\rangle}=\mathrm{e}^{(\mathrm{i} E t)}\left(\left(1-\frac{1}{2} \xi(t)^{*} \xi(t)\right)\left|\phi_{1}\right\rangle-\xi(t)\left|\phi_{2}\right\rangle\right)=\mathrm{e}^{(\mathrm{i} E t)} \mid \widetilde{\xi(t)\rangle} .
$$

The results (29) and (30) reveal the bi-normality and bi-overcompleteness of the family of time evolved states $\{|\xi ; t\rangle,|\widetilde{\xi ; t}\rangle\}$ of the phermionic oscillator system (4) - one has $\langle t ; \xi| \widetilde{\xi ; t\rangle}=1$, and

$$
1=\int \mathrm{d} \xi^{*} \mathrm{~d} \xi|\xi ; t\rangle \widetilde{\langle t ; \xi}=\int \mathrm{d} \xi^{*} \mathrm{~d} \xi|\widetilde{\xi ; t}\rangle\langle t ; \xi| .
$$

We observe that here the time evolved states $|\xi ; t\rangle$ and $\widetilde{|\xi ; t\rangle}$ differ from coherent states $|\xi(t)\rangle$ and $\widetilde{|\xi(t)\rangle}$ only in phase factors. 


\section{Concluding Remarks}

In this paper, we have constructed phermionic coherent states for the single phermionic oscillator. We have shown that these coherent states satisfy the usual properties of the coherent states: a) continuity of labelling, b) the resolution of identity, c) the temporal stability.

\section{References}

[1] Bagchi B. and Quesne C., Creation and Annihilation Operators and Coherent States for the PT-Symmetric Oscillator, Mod. Phys. Lett. A 16 (2001) 2449-2455.

[2] Cahill K. and Glauber R., Density Operators for Fermions, Phys. Rev. A 59 (1999) 1538-1555.

[3] Cherbal O., Drir M., Maamache M. and Trifonov D., Fermionic Coherent States for Pseudo-Hermitian Two-Level Systems, J. Phys. A: Math. Theor. 40 (2007) 1835-1844.

[4] Glauber R., Coherent and Incoherent States of the Radiation Field, Phys. Rev. 131 (1963) 2766-2788.

[5] Mostafazadeh A., Pseudo-Hermiticity Versus PT Symmetry: The Necessary Condition for the Reality of the Spectrum of a Non-Hermitian Hamiltonian, J. Math. Phys. 43 (2002) 205-214.

[6] Mostafazadeh A., Statistical Origin of Pseudo-Hermitian Supersymmetry and Pseudo-Hermitian Fermions, J. Phys. A: Math. \& Gen. 37 (2004) 10193-10207.

[7] Nikolov B. and Trifonov D., Dynamics of Generalized Coherent States I. Exact and Stable Evolution, Commun. JINR E2-81-797, Dubna, 1981.

[8] Ohnuki J. and Kashiwa T., Coherent States of Fermi Operators and the Path Integral, Prog. Theor. Phys. 60 (1978) 548-564.

[9] Perelomov A., Generalized Coherent States and their Applications, Springer, Berlin 1986.

[10] Roy B. and Roy P., Coherent States of Non-Hermitian Quantum Systems, Phys. Lett. A 359 (2006) 110-113.

[11] Trifonov D., Generalized Intelligent States and Squeezing, J. Math. Phys. 35 (1994) 2297-2308. 
[12] Zhang W., Feng D. and Gilmore R., Coherent States: Theory and Some Applications, Rev. Mod. Phys. 62 (1990) 867-927.

Omar Cherbal

Theoretical Physics Laboratory

Faculty of Physics USTHB

B.P 32 El-Alia, Bab Ezzouar

16111 Algiers

ALGERIA

E-mail address: ocherbal @yahoo.fr

Mahrez Drir

Theoretical Physics Laboratory

Faculty of Physics USTHB

B.P 32 El-Alia, Bab Ezzouar

16111 Algiers

ALGERIA 